\title{
Journal of Yoga, Physical Therapy and Rehabilitation
}

\section{Postural Control in Preschool Children with Developmental Coordination Disorder, in Sitting Position During a Functional Task}

\author{
Cristiana Isabel André Mercê ${ }^{*}$, Marco António Colaço Branco², Ana Paula de Lemos Teixeira e Seabra ${ }^{3}$, David Paulo Ramal- \\ heira Catela ${ }^{1}$ \\ ${ }^{1}$ Sport Sciences School of Rio Maior (ESDRM), Polytechnic Institute of Santarém (IPSantarém); IPSantarem Research Unit (UI-IPS) - \\ Applied Psychology; Life Quality Research Centre (CIEQV) - Motor Behaviour, Portugal
}

${ }^{2}$ Sport Sciences School of Rio Maior (ESDRM), Polytechnic Institute of Santarém (IPSantarém); IPSantarem Research Unit (UI-IPS); Interdisciplinary Centre for the Study of Human Performance (CIPER), University of Lisbon (ULisbon), Portugal

${ }^{3}$ Sport Sciences School of Rio Maior (ESDRM), Polytechnic Institute of Santarém (IPSantarém); IPSantarem Research Unit (UI-IPS) Applied Psychology, Portugal

"Corresponding author: Cristiana Isabel André Mercê, Sport Sciences School of Rio Maior (ESDRM), Applied Psychology- IPSantarem Research Unit (UI-IPS), Motor Behaviour-Life Quality Research Centre (CIEQV), Portugal. Tel: +351911955398; Email: cristianamerce@esdrm.ipsantarem.pt

Citation: Mercê C, Branco M, e Seabra A, Catela D (2018) Postural Control in Preschool Children with Developmental Coordination Disorder, in Sitting Position During a Functional Task. Yoga Phys Ther Rehabil: YPTR-157. DOI: 10.29011/ ISSN: 2577-0756. 000057

Received Date: 13 April, 2018; Accepted Date: 04 May, 2018; Published Date: 14 May, 2018

\begin{abstract}
The developmental coordination disorder is a motor disorder that affects 5 to $6 \%$ of children at school-age. The postural control deficit is one of the most prevalent problems affecting 73 to $87 \%$ of these children. The present research aims to analyze and compare postural control in probable (p-DCD) and at- risk of developmental coordination disorder children and in typical children in a sitting position during a functional task. p-DCD children were tendentially less recurrent, less periodic, simpler and more regular. These children oscillated more and faster in conditions with visual information; with a visual focus they had more stability and oscillated less and slowler campared to the baseline; without visual information they reduced their oscillations and velocity and become less recurrent, periodic, stable and simpler, possibly freenzing more degrees of freedom in order to respond to absence of external information. p-DCD seem to be more dependent on external stimulus like visual information to auto organize their own balance. The greater the task's complexity, the lesser and slower their oscillations were but also more recurrent and periodic. Despite oscillating, more and faster in all conditions and being tendentially more recurrent and periodic, in risk children revealed a behaviour pattern similar to typical in both variables. $\mathrm{p}-\mathrm{DCD}$, at-risk and typical children reveal the same manner of action without visual information, less and slower oscillations. Most likely, the problem with p-DCD is not in motor control, but on perception-action cycles' effectivness; and, where stimulation must be focused.
\end{abstract}

Keywords: Children; DCD; Functional Task; Postural Control; Sitting

\section{Introduction}

The Developmental Coordination Disorder (DCD) is a motor disorder without neural compromising identified and recognized by the Diagnostic and Statistical Manual of Mental Disorders (DSM) [1]. DCD affects 5-6\% of children within school-age, and it is referenced for impairment in fine and/or global coordination development, difficulty in motor control and learning, and in the acquisition of new motor skills [2,3]. This is a chronic disorder [4] which ethology is still not clear, one of the possibilities consists of a sensory integration deficit [5]. Motor impairment in DCD children varies in severity and nature [2], these are a heterogeneous group, as they can reveal only part of the symptoms as opposed to all simultaneously [5].

One of the most employed tools to diagnose DCD is the MABC-2 battery test (Pearson, United Kingdom), which allows 


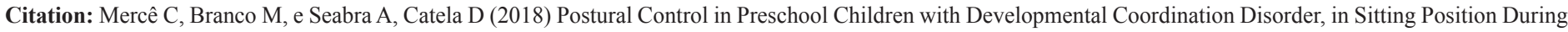
a Functional Task. Yoga Phys Ther Rehabil: YPTR-157. DOI: 10.29011/ ISSN: 2577-0756. 000057

to identify and describe the motor impairment in children [6]. By scoring the tests, the battery helps to identify if the children have probable DCD; are at-risk of developing DCD, which is a transition zone where the child does not have the disorder but have motor impairments; or, he/she is in a typical motor development zone. It is important to note that DCD should be diagnosed by a multidisciplinary team of professionals qualified to examine the specific criteria for the disorder [7], if it does not occur we can just talk about a probable DCD (p-DCD).

To provide the best possible intervention and therapy, first, it is necessary to understand the disorder in depth and how it triggers the problem that we want to lessen. One of the most prevalent problems is the Postural Control (PC) deficit, which affects 73 to $87 \%$ of the DCD children [8]. This impairment affects the daily life of the child since it is crucial for all daily tasks, like walking, running or playing. To understand more deeply how balance in DCD children evolves and differs from typical it is necessary to disturb the system and force it to reorganize itself, to analyze it and understand how it reacts to different stimulus. To analyze PC closer to the children's daily life it would be interesting to introduce a functional task which they can replicate in their daily life [9].

Bearing in mind that the DCD children could have a sensory integration deficit, the problem in PC could reside in its own process. So, to study PC it should be used methods that in spite of only quantifying movement, they can also analyze its quality and how the PC evolve over time. Nonlinear methods have recently proven that they can provide information that other methods cannot, namely, about the quality of movement and how the movement is controlled by the system as time goes by [10]. These methods are sensitive to small improvements and alterations in postural control over time and can also reveal significant differences between typical and delayed development in children with motor disabilities (e.g. cerebral palsy) [11-13].

The present research aims to analyze and compare PC in p-DCD, at-risk and typical children during a functional task in sitting position, in order to, if possible, identify clues for a most suitable intervention in the improvement of PC in p-DCD children.

Considering the necessity to evaluate the quality movement, besides the linear methods including posturography variables like total distance, amplitude and velocity of the points in study, it were also used nonlinear methods including Recurrence Quantification Analysis (RQA), Lyapunov Exponent (LyE) and sample entropy (SampEn). RQA is a non-linear and multidimensional technique that reconstructs the temporal series in the space to verify the recurrent points also known as neighbor points [14]. This method provides several variables that can describe the system, allowing its analysis through: i) percent of recurrence (\%RECUR or $\%$ REC) - percentage of recurrent points that fall in the radius; ii) maxline - the biggest length of the diagonal lines, which specifies a measurement of the global stability; iii) mean line - mean of the diagonal lengths, where a bigger mean line implies that the system is enter in longer deterministic states, so it consists of a periodicity measurement; iv) entropy - measurement of the complexity system, the higher the entropy the higher the complexity $[14,15]$.

The Lyapunov Exponent (LyE) is another nonlinear technique that can detect the presence of chaos in the system and has been used to analyze biological systems. This variable measures the rate of how nearly orbits converge or diverge in the state space. In periodic signals, the orbits will not diverge or converge so the LyE would be zero because the trajectories in the state space are completely overlapped. If the orbits diverge, the system is exploring an exponential growth, the LyE would be superior to zero. If the orbits converge the system is exploring an exponential decay, and the LyE would be inferior to zero. A positive LyE indicates chaos in the system, the larger the LyE the bigger the instability [16].

The SampEn is an improvement of approximate entropy [17] which designate that if the data are predictable or not. A lower sample entropy (SampEn) means more regularity in the system. For a correct function of SampEn, the data should be larger than 200 data points, the longer the better, and we could not compare time series with different lengths [18].

\section{Methods}

\section{Sample}

The study took place in three Portuguese kindergartens in the region of Rio Maior. The identification of children with probable DCD and at- risk was conducted according to MABC-2 protocol, band 1 [6]; and identified by an expert panel of three experts in motor behavior. It was conducted the informed consent of the parents and the assent of the participants, being that the will of children was always respected.

It was included 14 children of both gender, and a mean age of $3.98 \pm 0.24$ years old. For the two probable DCD and for the five at- risk children, 7 children with typical motor development were paired. The pairing was made by gender, age, kindergarten, and MABC-2's scores, being that all typical children had a percentile score higher than 25 [19]. It was only considered children with 3 and 4 years due to that the earlier the intervention and diagnosis the better the results [20]. Children that violated the criteria of DSMIV for DCD, like intellectual disability, visual impairment and neurological condition that affects movement, were not included [1].

\section{Tasks}

Children completed five tasks (conditions), always in the following order: i) just being seated (SEO), the child was seated on a bench forming a $90^{\circ}$ angle at the knee, with both feet on the floor, without restrictions referring to arms and hands positions [21]; ii) 


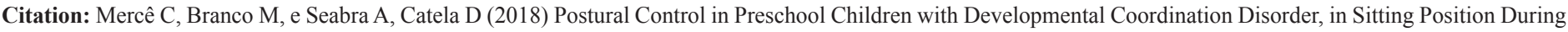
a Functional Task. Yoga Phys Ther Rehabil: YPTR-157. DOI: 10.29011/ ISSN: 2577-0756. 000057

same as in first condition, but with the eyes closed (SEC), to blindfold the children it was used a cloth; iii) the child observed a modulation of a ball with plasticine made by the researcher in front of him/her and at his/her eye level (SD), slowly and roughly; iv) the child molded the plasticine by himself to make a ball (DEO) no restriction was made to time, arms and hands movements, and using the legs to support the action [21]; v) same as in iv), but with the eyes blindfolded (DEC), see figure 1. Before starting, the researcher explained and reinforced to the child that he/she did not have to think if he/she was doing well or not, what mattered was to simply do the task.
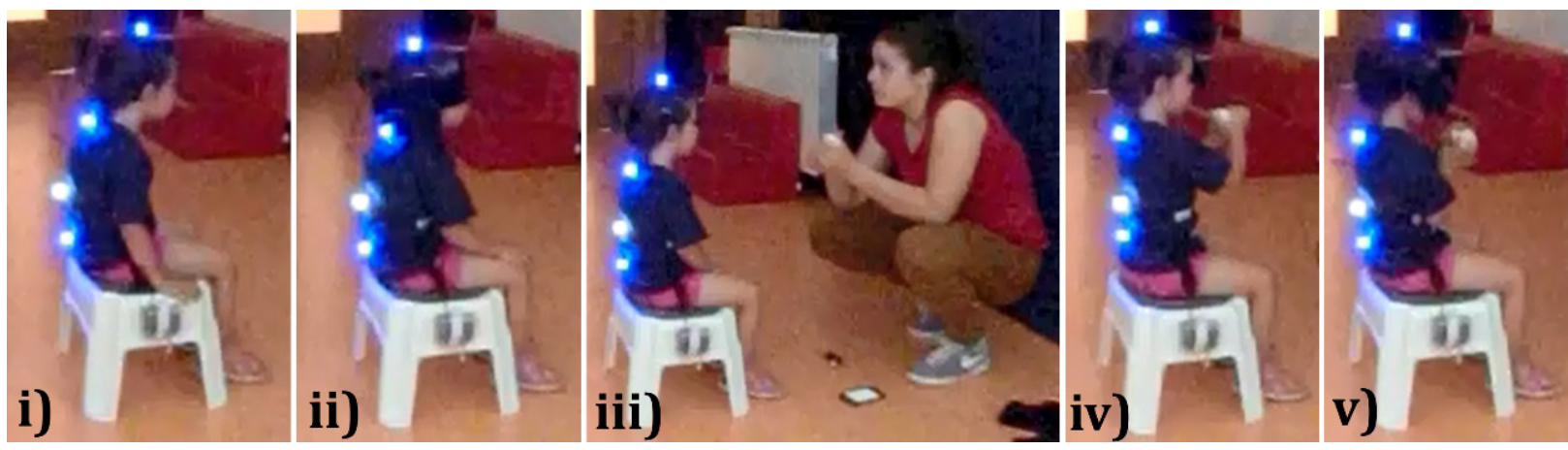

Figure 1: Experimental setup and conditions, from i) to v) (left to right).

With different conditions it was pursued different stimulation purposes. In condition ii) it was removed visual information, with the purpose of having information on the role of vision in a baseline task, simply being seated. With condition iii) it was introduced a functional visual focus [e.g. 9]; different from condition i), in this condition the visual information was necessary to observe an action performed by another person, parallel to its role in postural control. In condition iv), the child had to use his/her visual information to complete a functional task, but, simultaneously, he/she needed to control his/her posture in order to support the task in hand, possibly with an additional appeal to proprioceptive information. Finally, in condition v), with the removal of visual information, the child had to resort to proprioceptive (and haptic) information, in order to preserve postural control and to detect the evolution of the task to be completed in plasticine molding (Table 1).

\begin{tabular}{|c|c|c|c|c|c|}
\hline Condition & i) & ii) & iii) & iv) \\
\hline Description - Abbreviation & Sitting eyes open - SEO & $\begin{array}{c}\text { Sitting eyes closed } \\
- \text { SEC }\end{array}$ & See doing - SD & $\begin{array}{c}\text { Doing eyes } \\
\text { open - DEO }\end{array}$ & $\begin{array}{c}\text { Doing eyes } \\
\text { closed - DEC }\end{array}$ \\
\hline Stimulus & Baseline & No VI & VF & FT and no VI \\
\hline \multicolumn{2}{|c|}{ No VI - No Visual Information; VF - Visual Focus; FT - Functional Task } \\
\hline
\end{tabular}

Table 1: Study conditions and purpose.

The order and the preservation of the sequence of the tasks were based on the progressive difficulty of them, and on the need to initially have baseline references unaffected by functional task interferences. To see what was meant to be done, before doing it, also helped the children to understand the task to be done. It would be possibly to alternate the order of presentation of conditions iv) and v), however, it would be possible that the inversion of this order be too complex for the children with probable DCD, leading them to avoid the task or to abandon the study.

\section{Data Collection}

Data were collected for anatomic points: vertex $(\mathrm{V}$, point that represent the head movements), and cervical 7 (C7, that represent the trunk movements). Two high definition cameras,
Casio model Exilim Ex-ZR200, recording at $240 \mathrm{~Hz}$, were placed perpendicularly to frontal and sagittal plane of the children [22]. The points were identified using led markers.

The filming in conditions i), ii) and iii) had a 30 second duration, the maximum time for balance tests in MABC-2 [6]. In conditions iv) e v) the filming lasted the time that the child took to create the plasticine ball. The beginning of the data collection matched when the child grabbed the ball, and the end matched when the child informed the researcher that he/she had finished it.

\section{Data Treatment}

Kinematic analysis was performed with Ariel Performance Analysis System (Ariel Dynamics, Inc., version 2003), and 


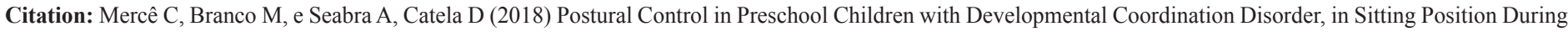
a Functional Task. Yoga Phys Ther Rehabil: YPTR-157. DOI: 10.29011/ ISSN: 2577-0756. 000057

nonlinear analysis was performed through Matlab (Mathworks, Inc., Massachusetts, USA).

The data collection had a duration of 30 seconds, however in some cases, children did not take this long to complete the task. So, it was selected the smaller time series of 3600 data points, and it was considered just the first 3600 data points off all time series, so all of them could be under the same conditions [18].

In order to identify the data ideal frequency, it was performed the power spectrum in time series [23], in which was identified a frequency of $60 \mathrm{~Hz}$. So, it was performed a down sample of the data, obtaining a time series with 900 data points, all of these steps were performed in Matlab.

For nonlinear treatment there were not applied any filters in order to not alter nonlinear measurements [12]. For linear treatment it was applied a digital filter with a $12 \mathrm{~Hz}$ cutoff. All linear and nonlinear variables were performed in Matlab.

\section{Statistical Analysis and Error Measurement}

For statistical analysis and error measurement it was used the Statistical Package for the Social Sciences (IBM Corp. SPSS Statistics for Windows, version 24). It was used descriptive statistics to analyze the axis of anterior-posterior movements [24] for total distance (TT-AP), the Antero-Posterior (A-AP) amplitude, and Antero-Posterior Mean Velocity (MV-AP); by group, anatomical point and condition.

APAS application error was measured. The dynamic error was considered for each point, $\mathrm{V}$ and $\mathrm{C} 7$, and for each motion plane, $\mathrm{X}$ is the axis that correspond to anteroposterior motion (AP), $\mathrm{Y}$ the axis for vertical motion (Vert) and $\mathrm{Z}$ for Mediolateral motion (ML). To evaluate the dynamic error, it was performed twice the automatic digitalization for the same data with the same volume calibration scanning. The displacement outputs of the two digitalization's were analyzed with descriptive analysis calculating the mean, standard deviation and mean differences in order to obtain the average error (Table 2). It was also performed the Inter Correlation Coefficient (ICC), that is a statistic technique which measures the values reliability of two or more measures, the ICC values less than 0.5 are considered to be poor, between 0.5 and 0.75 moderate, between 0.75 and 0.9 good, and greater than 0.9 excellent [25]. In present data it was verified 1 moderate ICC value, 1 good and 4 excellent. The transverse plan was the less reliable with moderate and good values, while frontal and sagittal planes revealed excellent values (Table 3 ).

\begin{tabular}{|c|c|c|c|c|c|}
\hline \multirow{2}{*}{ Point / Type of Motion } & \multicolumn{2}{|c|}{ Mean } & \multicolumn{2}{|c|}{ Standard Deviation } & \multirow{2}{*}{ Average Error } \\
\hline & $1^{\text {st }}$ & $2^{\text {nd }}$ & $1^{\text {st }}$ & $2^{\text {nd }}$ & \\
\hline $\mathrm{C} 7 / \mathrm{AP}$ & 521.3 & 522.8 & 3.46 & 3.45 & -1.5 \\
\hline C7 / Vert & 606.1 & 603 & 3.21 & 3.22 & 3.12 \\
\hline $\mathrm{C} 7 / \mathrm{ML}$ & 576.3 & 575.9 & 14.77 & 14.8 & 0.4 \\
\hline $\mathrm{V} / \mathrm{AP}$ & 496.6 & 498 & 5 & 5.03 & -1.39 \\
\hline V / Vert & 817.1 & 814.6 & 3.92 & 3.95 & 2.47 \\
\hline $\mathrm{V} / \mathrm{ML}$ & 511 & 510.2 & 21.82 & 21.9 & 0.86 \\
\hline
\end{tabular}

Table 2: Average Error for points and type of motion, displacement in $\mathrm{mm}$.

\begin{tabular}{|c|c|c|c|c|}
\hline \multicolumn{2}{|c|}{} & \multicolumn{3}{|c|}{ Type of Motion } \\
\cline { 3 - 5 } \multicolumn{2}{|c|}{} & AP & Vert & ML \\
\hline Points & C7 & 0.914 & 0.679 & 1 \\
\hline & V & 0.963 & 0.836 & 0.999 \\
\hline
\end{tabular}

Table 3: ICC for points and type of motion.

For data analysis it was performed descriptive statistics with calculation of mean and standard derivation to characterize the sample, linear and nonlinear data. For both linear and nonlinear data, the statistical test Kruskal-Wallis was applied to compare the results between groups for the same condition and point. Bonferroni correction was considered and a level of significance of $\mathrm{p}=0.05$, two-tailed was adopted.

\section{Results and Discussion}

\section{Posturography Results}

No significant differences were found between groups for the same condition and point studied, see Kruskall-Wallis test results in table 4 below, due to the Bonferroni correction for being significantly different $\mathrm{p}$ should be less than 0.01 . 


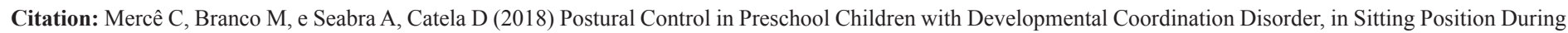
a Functional Task. Yoga Phys Ther Rehabil: YPTR-157. DOI: 10.29011/ ISSN: 2577-0756. 000057

\begin{tabular}{|c|c|c|c|c|c|c|c|}
\hline \multirow{7}{*}{ Point } & \multirow[t]{2}{*}{ Condition } & \multicolumn{2}{|c|}{ Total Distance (TT-AP) } & \multicolumn{2}{|c|}{ Amplitude (A-AP) } & \multicolumn{2}{|c|}{ Mean Velocity (MV-AP) } \\
\hline & & H(2) & $\mathbf{P}$ & $\mathbf{H}(\mathbf{2})$ & $\mathbf{p}$ & $\mathbf{H}(2)$ & $\mathbf{P}$ \\
\hline & SEO & 5.15 & 0.075 & 4.033 & 0.133 & 5.18 & 0.075 \\
\hline & SEC & 6 & 0.05 & 8.501 & 0.014 & 6 & 0.05 \\
\hline & SD & 1.187 & 0.552 & 1.494 & 0.474 & 1.187 & 0.552 \\
\hline & DEO & 0.821 & 0.663 & 1.885 & 0.39 & 0.821 & 0.663 \\
\hline & $\mathrm{DEC}$ & 3.159 & 0.206 & 0.168 & 3.157 & 0.206 & 3.159 \\
\hline \multirow{5}{*}{$\mathrm{C} 7$} & SEO & 6.913 & 0.032 & 6.913 & 0.032 & 6.913 & 0.032 \\
\hline & SEC & 2.976 & 0.226 & 3.571 & 0.168 & 2.976 & 0.226 \\
\hline & $\mathrm{SD}$ & 2.162 & 0.339 & 3.021 & 0.221 & 2.162 & 0.339 \\
\hline & DEO & 0.05 & 0.975 & 0.501 & 0.778 & 0.05 & 0.975 \\
\hline & DEC & 3.113 & 0.211 & 7.548 & 0.023 & 3.113 & 0.211 \\
\hline
\end{tabular}

Table 4: Comparisons for linear data between groups for the same condition and point.

For $\mathrm{p}$-DCD the conditions involving opened eyes (SEO and DEO) are the ones where they oscillated more (in total and in amplitude) and faster, contrasting to at- risk and typical children where despite oscillating more and faster in DEO they oscillate less and more slowly in SEO, see table 5. Previous studies, also revealed that $\mathrm{PC}$ children oscillate more in AP (anteroposterior) direction than typical ones during sitting position with eyes open [23]. When a visual focus of attention was introduced (SD condition) p-DCD children oscillated less and more slowly compared to the baseline condition. Possibly, for p-DCD the focus on researcher manipulation worked as a visual anchor that in some way altered their postural control. However, this slowing is even more notorious when we remove visual information (SEC and DEC conditions). Passing the condition of SEO to SEC p-DCD children decrease their oscillations and velocity while at- risk and typical increase. Moreover, when we removed visual information during the task, passing of DEO to DEC, p-DCD children decrease their oscillations and velocity once more, even noticing that all groups that reduced these variables p-DCD are the ones that revealed the lowest values. In these cases, we cannot attribute changings in postural oscillations to an external visual focus, it is more like they freeze degrees of freedom, in order to respond to absence of external information; left to their internal information, these children became more conservative in their postural oscillations, as a necessary condition to preserve postural stability and to perform the task in hand. The greater the complexity of the task, the lesser and slower the oscillations are.

At- risk children revelead the highest TD-AP, A-AP and MVAP, in all conditions, except for SEO. It could be that, the transition zone where at- risk children may be, in terms of postural control, forces them to try to explore solutions, which result in more and faster body oscillations (in total and amplitude).

Despiste being the group that oscillated more and faster under all conditions, at- risk children revealed a pattern of behaviour similar to typical children. For the two groups in the less complex conditions, SEO e SEC, they oscillate less and more slowly, and for more complex conditions that envolve a functional task, DEO e DEC, they oscillate more and faster.

It is interesting to note that, similarly to p-DCD, the removal of visual information during a functional task makes children oscillate less and more slowly, compared to the same condition with eyes open. So in general, DCD, at risk and typical developing children reveal the same way of action when visual information removal occurs during a functional task, less and slower oscillations; thus, DCD children have the same qualitative postural mechanisms as typical children, although with different quantitative outputs. It might mean, that the problem of DCD children is not in modes of motor control, but in perception-action cycles' effectiveness [26], where stimulation must be focused. 


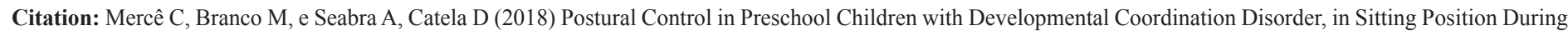
a Functional Task. Yoga Phys Ther Rehabil: YPTR-157. DOI: 10.29011/ ISSN: 2577-0756. 000057

\begin{tabular}{|c|c|c|c|c|c|c|}
\hline \multicolumn{2}{|c}{} & SEO & SEC & SD & DEO & DEC \\
\hline \multirow{4}{*}{ Total Distance (TT-AP) } & $\mathrm{p}-\mathrm{DCD}$ & $1219.41 \pm 974.62$ & $493.93 \pm 9.72$ & $885.9 \pm 244.01$ & $1131.78 \pm 156.73$ & $717.52 \pm 9.27$ \\
\cline { 2 - 7 } & $\mathrm{R}$ & $587.75 \pm 368.36$ & $1217.93 \pm 933.45$ & $1234.64 \pm 755.29$ & $1676.41 \pm 458.59$ & $1341.23 \pm 731.94$ \\
\cline { 2 - 7 } & $\mathrm{T}$ & $351.48 \pm 264.98$ & $403.91 \pm 199.74$ & $742.24 \pm 329.89$ & $1462.46 \pm 698$ & $812.03 \pm 205.72$ \\
\hline \multirow{3}{*}{ Amplitude (A-AP) } & $\mathrm{p}-\mathrm{DCD}$ & $84.91 \pm 62.11$ & $36.3 \pm 9.72$ & $67.56 \pm 11.3$ & $74.7 \pm 10.39$ & $52.22 \pm 1.98$ \\
\cline { 2 - 7 } & $\mathrm{R}$ & $40.51 \pm 24.14$ & $88.73 \pm 52.86$ & $85.21 \pm 45.07$ & $111.99 \pm 24.18$ & $95.17 \pm 45.37$ \\
\cline { 2 - 7 } & $\mathrm{T}$ & $26.48 \pm 15.31$ & $29.05 \pm 12.09$ & $52.5 \pm 24.23$ & $99.81 \pm 36.79$ & $56.89 \pm 12.9$ \\
\hline \multirow{3}{*}{ Mean Velocity (MV-AP) } & $\mathrm{p}-\mathrm{DCD}$ & $20.32 \pm 16.24$ & $8.23 \pm 2.94$ & $14.77 \pm 4.07$ & $18.86 \pm 2.61$ & $11.96 \pm 0.16$ \\
\cline { 2 - 7 } & $\mathrm{R}$ & $9.8 \pm 6.14$ & $20.3 \pm 15.56$ & $20.58 \pm 12.59$ & $27.94 \pm 7.64$ & $22.35 \pm 12.2$ \\
\cline { 2 - 7 } & $\mathrm{T}$ & $5.86 \pm 4.42$ & $6.73 \pm 3.3$ & 12.375 .5 & $24.37 \pm 11.63$ & $13.53 \pm 3.43$ \\
\hline
\end{tabular}

Table 5: Descriptive statistics of posturography variables for vertex point

Both in vertex and in C7 (Table 6), the condition of SEO, which represents the baseline, promoted the biggest and fastest oscillations inside p-DCD group, and also the smaller and more slowly inside the at- risk and typical children. Looking at p-DCD group, the patterns identified in $\mathrm{V}$ are also present in $\mathrm{C} 7$, the condition where p-DCD oscillated less and more slowly was the SEC. Once again, when we removed visual information from SEO to SEC, p-DCD began to oscillate less and more slowly when at- risk and typical increased their values. When we moved from DEO to DEC, $\mathrm{p}-\mathrm{DCD}$ also reduced oscillations and velocity. This way, the visual information seems to be an important part of p-DCD postural control, when the difficulty increased with no visual information, it conducted to smaller and slower oscillations. Following this line of thought, it may be strange to see that DEC revealed bigger and faster oscillation than in SEC, supposedly doing a functional task blindfolded is harder that just being seated with eyes closed. Nonetheless, the data makes us believe that p-DCD are more dependent from external stimulus to self- organize their own balance. So, comparing DEC and SEC, p-DCD children had more external information with proprioceptive information about the mold of the ball, perhaps, the simple fact of doing a functional task can help them to manage their posture. If this is true, we should rethink our intervention in these children and focus on functional tasks.

At -risk and typical children also continued to reveal a similar pattern, as shown in vertex. Again, the conditions where children oscillated less and more slowly were the non-manipulation conditions, SEO and SEC. In conditions that involve the functional task, DEO e DEC, children started to oscillate more and faster, compared to the non-manipulation conditions. The removal of visual information, during the functional tasks also decreased $\mathrm{C} 7$ oscillations and velocity in typical children, and, at- risk children revealed similar values in DEO and DEC.

It is interesting to refer, that for all groups, in all points and in all conditions the head point always oscillated more and faster than the point in C7. So, it seems that the control of the head and the trunk is coordinated, in the sense of intersegmental coordination, independently of the pattern of motor development, which has an observable effect in the quantitative pattern of oscillations but not in its qualitative one. However, we need a different method to admit this last hypothesis, because with these traditional methods we cannot observe the true pattern of oscillation in each of these groups of children.

\begin{tabular}{|c|c|c|c|c|c|c|}
\hline \multicolumn{2}{c}{} & SEO & SEC & SD & DEO & DEC \\
\hline \multirow{3}{*}{ Total Distance (TT-AP) } & $\mathrm{p}-\mathrm{DCD}$ & $697.47 \pm 677.94$ & $262.56 \pm 106.03$ & $606.52 \pm 73.35$ & $544.31 \pm 154.6$ & $520.56 \pm 143.59$ \\
\cline { 2 - 7 } & $\mathrm{R}$ & $373.11 \pm 185.57$ & $536.72 \pm 406.86$ & $806.89 \pm 622.7$ & $691.44 \pm 440.96$ & $693.93 \pm 295.03$ \\
\cline { 2 - 7 } & $\mathrm{T}$ & $128.4 \pm 68.77$ & $147.83 \pm 48.75$ & $358.38 \pm 207.39$ & $534.87 \pm 299.26$ & $415.81 \pm 102.35$ \\
\hline \multirow{3}{*}{ Amplitude (A-AP) } & $\mathrm{p}-\mathrm{DCD}$ & $51.13 \pm 48.55$ & $20.86 \pm 5.2$ & $55.06 \pm 2.27$ & $46.93 \pm 13.57$ & $48.36 \pm .16$ \\
\cline { 2 - 7 } & $\mathrm{R}$ & $27.78 \pm 12.46$ & $37.74 \pm 28.21$ & $54.41 \pm 43.59$ & $56.47 \pm 36.22$ & $55.72 \pm 21.51$ \\
\cline { 2 - 7 } & $\mathrm{T}$ & $9.59 \pm 5.28$ & $10.85 \pm 2.64$ & $28.97 \pm 16.44$ & $40.54 \pm 22.49$ & $30.57 \pm 7.86$ \\
\hline \multirow{3}{*}{ Mean Velocity (MV-AP) } & $\mathrm{p}-\mathrm{DCD}$ & $11.62 \pm 11.3$ & $4.38 \pm 1.77$ & $10.11 \pm 1.22$ & $9.07 \pm 2.58$ & $8.68 \pm 2.39$ \\
\cline { 2 - 7 } & $\mathrm{R}$ & $6.22 \pm 3.1$ & $8.95 \pm 6.78$ & $13.45 \pm 11.05$ & $11.52 \pm 7.35$ & $11.57 \pm 4.92$ \\
\cline { 2 - 7 } & $\mathrm{T}$ & $2.14 \pm 1.15$ & $2.46 \pm 0.1$ & $5.97 \pm 3.46$ & $8.91 \pm 4.99$ & $6.93 \pm 1.71$ \\
\hline
\end{tabular}

Table 6: Descriptive statistics of posturography variables for C7 point. 


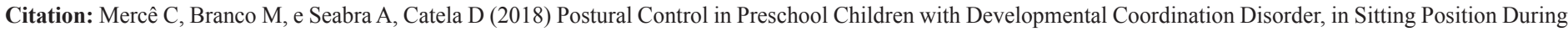
a Functional Task. Yoga Phys Ther Rehabil: YPTR-157. DOI: 10.29011/ ISSN: 2577-0756. 000057

\section{Nonlinear Results}

There were found no significant differences between the nonlinear data for the same condition and point (Table 7).

\begin{tabular}{|c|c|c|c|c|c|c|c|c|c|c|c|c|c|}
\hline \multirow{2}{*}{ Point } & \multirow{2}{*}{ Condition } & \multicolumn{2}{|c|}{ REC } & \multicolumn{2}{|c|}{ Mean line } & \multicolumn{2}{|c|}{ Maxline } & \multicolumn{2}{|c|}{ LyE } & \multicolumn{2}{|c|}{ Entropy } & \multicolumn{2}{|c|}{ Sample Entropy } \\
\hline & & $\mathbf{H}(2)$ & $\mathbf{p}$ & $\mathbf{H}(2)$ & $\mathbf{p}$ & $\mathbf{H}(2)$ & $\mathbf{p}$ & $\mathbf{H}(2)$ & $\mathbf{p}$ & $\mathbf{H}(2)$ & $\mathbf{p}$ & $\mathbf{H}(2)$ & $\mathbf{P}$ \\
\hline \multirow{5}{*}{$\mathrm{V}$} & SEO & 1.278 & 0.528 & 3.142 & 0.208 & 4.228 & 0.121 & 1.673 & 0.433 & 2.045 & 0.36 & 2.389 & 0.303 \\
\hline & SEC & 0.142 & 0.931 & 0.501 & 0.778 & 1.641 & 0.44 & 0.873 & 0.646 & 4.805 & 0.09 & 4.128 & 0.127 \\
\hline & SD & 0.507 & 0.776 & 1.193 & 0.551 & 1.082 & 0.582 & 0.005 & 0.998 & 1.96 & 0.375 & 0.124 & 0.94 \\
\hline & DEO & 5.902 & 0.052 & 5.04 & 0.08 & 3.397 & 0.183 & 0.056 & 0.972 & 5.04 & 0.05 & 1.727 & 0.422 \\
\hline & DEC & 1.736 & 0.42 & 2.731 & 0.255 & 0.217 & 0.897 & 2.591 & 0.274 & 3.368 & 0.186 & 3.104 & 0.212 \\
\hline \multirow{5}{*}{$\mathrm{C} 7$} & SEO & 2.16 & 0.34 & 0.954 & 0.621 & 0.954 & 0.621 & 0.948 & 0.623 & 1.151 & 0.469 & 3.433 & 0.18 \\
\hline & SEC & 0.05 & 0.975 & 1.682 & 0.431 & 1.689 & 0.43 & 2.16 & 0.34 & 1.64 & 0.44 & 4.521 & 0.104 \\
\hline & SD & 1.216 & 0.545 & 0.547 & 0.761 & 4.165 & 0.125 & 0.331 & 0.848 & 0.73 & 0.694 & 2.179 & 0.336 \\
\hline & DEO & 0.351 & 0.839 & 0.136 & 0.934 & 0.079 & 0.961 & 0.24 & 0.887 & 0.273 & 0.873 & 0.691 & 0.708 \\
\hline & DEC & 0.828 & 0.661 & 6.816 & 0.033 & 1.733 & 0.42 & 3.593 & 0.166 & 5.902 & 0.052 & 6.764 & 0.034 \\
\hline
\end{tabular}

Table 7: Comparisons for nonlinear data between groups for the same condition and point.

Considering the nonlinear data by anatomical points (Table 8 and Table 9), it's possible to verify that for the same condition between groups, the p-DCD revealed the lowest value for REC, mean line, entropy and SampEn in all conditions except mean line in SEC and for SampEn in SEC and SD. P-DCD also revealed the lowest maxline in the conditions involving a task DEO and DEC in V. Possibly due to having postural control problems p-DCD children become less recurrent (lowest REC), less periodic (lowest mean line) and also simpler (lowest entropy). At- risk children in vertex revealed the highest REC, mean line and maxline values except mean line in SEC and maxline in SEO and SEC, and also revealed the highest values of entropy for SEC and SD. For C7 point atrisk children revealed the highest values of REC, mean line except in SD and also entropy except in SD. Considering that the linear data appoint at risk children as the ones with more displacement, amplitude and velocity in vertex, the nonlinear demonstrated that these children are also the most recurrent and tend to be the most periodic (mean line). Due to being in a transition zone at- risk child may become more recurrent and periodic to compensate their difficulties.

Therefore, considering the data related to vertex (Table 8) and observing the data inside the same group, p-DCD had the lower values of REC, mean line, maxline and entropy in conditions involving a functional task DEO and DEC. Which is consistent with linear data to the decrease in displacement, amplitude and velocity compared to the baseline conditions SEO and SEC. Surprisingly, for at- risk and typical children the removal of visual information SEO to SEC and DEO to DEC provoked a decrease in values of REC and mean line in both points, and reduced maxline and entropy in $\mathrm{V}$.

P-DCD children that had already revealed in linear data to oscillate less and slower in SEC condition, also revealed in nonlinear data to be more recurrent (higher REC in V and C7) and periodic (higher mean line in $\mathrm{V}$ ) in the same condition. So, the conditions in study with no visual information and less external information, compared to the others with the functional task which allowed proprioceptive information about the molding, was the one that originate less and slower oscillations but also the most recurrent and periodical ones. This could contribute to reinforce that the external information, like visual information, is very important to $\mathrm{p}$-DCD children. When external information is removed they restricted and reduced velocity in their oscillations which also became more recurrent and periodic. This increase of recurrence could be one more strategy for these children to compensate their balance problems.

Looking at LyE, and bearing in mind that a positive $\mathrm{LyE}$ indicates chaos in the system and the larger the LyE the bigger the instability [16], the condition with more stability for p-DCD in both points and at- risk for $\mathrm{V}$ was the one with a visual focus, $\mathrm{SD}$, probably the visual anchor of the researcher molding the ball provided more stability in the children. The less stable condition for all groups in $\mathrm{V}$ and $\mathrm{C} 7$ (except for at risk in C7) was DEC, which we considered initially to be the most difficult. This highest value of LyE in DEC condition was also accompanied by the smallest mean line values for all groups, in addition, remembering the linear data we also verified and decreased in amplitude and velocity of the oscillations compared to the same condition with eyes open for all children. So, probably the increased difficulty of the condition forced all children to oscillate less and slower, having more instability and being less periodic.

Bearing in mind, that entropy is a measure of predictability or regularity of the system the higher the entropy, the less regular the system is [27], p-DCD children revealed to be less regular in SEO for $\mathrm{V}$ and in SD for C7, and more regular in DEC for both 


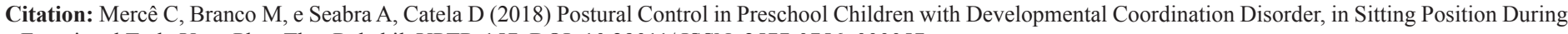
a Functional Task. Yoga Phys Ther Rehabil: YPTR-157. DOI: 10.29011/ ISSN: 2577-0756. 000057

points. This greater regularity in DEC contrasts with the smaller recurrence and periodicity already mentioned. A possible reason for this resided in the fact that entropy only oscillated a few decimals between conditions, we should probably rethink the calculating of entropy namely with a bigger time series.

\begin{tabular}{|c|c|c|c|c|c|c|}
\hline & & SEO & SEC & SD & DEO & DEC \\
\hline \multirow{3}{*}{ REC } & p-DCD & $.22 \pm .002$ & $.23 \pm .11$ & $.22 \pm .005$ & $.14 \pm .003$ & $.14 \pm 0.9$ \\
\hline & $\mathrm{R}$ & $.34 \pm .15$ & $.23 \pm .09$ & $.27 \pm .11$ & $.29 \pm .04$ & $.24 \pm .13$ \\
\hline & $\mathrm{T}$ & $.3 \pm .1$ & $.23 \pm .05$ & $.24 \pm .04$ & $.24 \pm .06$ & $.17 \pm .05$ \\
\hline \multirow{3}{*}{ Mean line } & p-DCD & $39.7 \pm 2.92$ & $60.96 \pm 38.77$ & $53.06 \pm 34.47$ & $32.9 \pm 11.27$ & $27.42 \pm 2.37$ \\
\hline & $\mathrm{R}$ & $101 \pm 63.55$ & $55.59 \pm 24.22$ & $53.37 \pm 18.52$ & $68.06 \pm 23$ & $49.45 \pm 26.58$ \\
\hline & $\mathrm{T}$ & $78.98 \pm 41.17$ & $58.08 \pm 23.82$ & $65.07 \pm 25.21$ & $56.97 \pm 11.87$ & $41.66 \pm 6.27$ \\
\hline \multirow{3}{*}{ Maxline } & p-DCD & $874,5 \pm 6.39$ & $868.5 \pm 2.12$ & $865.5 \pm 10.6$ & $852 \pm 4.24$ & $855 \pm 21.21$ \\
\hline & $\mathrm{R}$ & $865.4 \pm 9.0$ & $865 \pm 21.66$ & $867.7 \pm 12.21$ & $866 \pm 7.35$ & $863.4 \pm 7.77$ \\
\hline & $\mathrm{T}$ & $854.71 \pm 14.09$ & $853.86 \pm 18.15$ & $858 \pm 15.89$ & $864.57 \pm 11.93$ & $861.86 \pm 10.64$ \\
\hline \multirow{3}{*}{ LyE } & p-DCD & $9.04 \pm 6.07$ & $5.93 \pm 6.38$ & $3.07 \pm 3.07$ & $5.54 \pm .64$ & $10.86 \pm 4.67$ \\
\hline & $\mathrm{R}$ & $4.34 \pm 2.21$ & $4.37 \pm 3.85$ & $4.1 \pm 4.44$ & $6.77 \pm 3.85$ & $7.18 \pm 1.71$ \\
\hline & $\mathrm{T}$ & $4.7 \pm 4.07$ & $3.24 \pm 3.21$ & $3.67 \pm 3.64$ & $5.55 \pm 4.38$ & $5.77 \pm 2.92$ \\
\hline \multirow{3}{*}{ Entropy } & $\mathrm{p}-\mathrm{DCD}$ & $4.38 \pm 0.94$ & $4.22 \pm .035$ & $4.27 \pm .79$ & $4.04 \pm .42$ & $3.81 \pm .22$ \\
\hline & $\mathrm{R}$ & $4.99 \pm .57$ & $4.4 \pm .22$ & $4.63 \pm .36$ & $4.83 \pm .24$ & $4.43 \pm .57$ \\
\hline & $\mathrm{T}$ & $4.75 \pm .54$ & $4.6 \pm .27$ & $4.78 \pm .3$ & $4.7 \pm .23$ & $4.34 \pm .2$ \\
\hline \multirow{3}{*}{ Sample Entropy } & $\mathrm{p}-\mathrm{DCD}$ & $.003 \pm .004$ & $.002 \pm .00005$ & $.001 \pm .001$ & $0 \pm .00004$ & $.003 \pm .001$ \\
\hline & $\mathrm{R}$ & $.003 \pm .002$ & $.002 \pm .003$ & $.002 \pm .002$ & $0 \pm .00004$ & $.002 \pm .005$ \\
\hline & $\mathrm{T}$ & $.008 \pm .009$ & $.007 \pm .007$ & $.002 \pm .004$ & $.0002 \pm .0003$ & $.0016 \pm .001$ \\
\hline
\end{tabular}

Table 8: Descriptive statistical for nonlinear variables for vertex point.

\begin{tabular}{|c|c|c|c|c|c|c|}
\hline & & SEO & SEC & SD & DEO & DEC \\
\hline \multirow{3}{*}{ REC } & $\mathrm{p}-\mathrm{DCD}$ & $.17 \pm .03$ & $.24 \pm 1$ & $.23 \pm .03$ & $.21 \pm .01$ & $.13 \pm .09$ \\
\hline & $\mathrm{R}$ & $.31 \pm .23$ & $.25 \pm .08$ & $.31 \pm .09$ & $.29 \pm .21$ & $.18 \pm .02$ \\
\hline & $\mathrm{T}$ & $.24 \pm .06$ & $.23 \pm .08$ & $.29 \pm .1$ & $.21 \pm .06$ & $.15 \pm .04$ \\
\hline \multirow{3}{*}{ Mean line } & p-DCD & $33.27 \pm .82$ & $24.46 \pm 20.62$ & $72.87 \pm 31.78$ & $45.78 \pm 33.1$ & $15.4 \pm 6.27$ \\
\hline & $\mathrm{R}$ & $96.57 \pm 91.76$ & $70.22 \pm 48.36$ & $64.55 \pm 22.7$ & $60.96 \pm 10.94$ & $41.95 \pm 16.23$ \\
\hline & $\mathrm{T}$ & $53.9 \pm 26.5$ & $59.5 \pm 35.5$ & $70.43 \pm 35.01$ & $47.25 \pm 22.5$ & $29.41 \pm 6.82$ \\
\hline \multirow{3}{*}{ Maxline } & $\mathrm{p}-\mathrm{DCD}$ & $858 \pm 1414$ & $873 \pm 21.21$ & $846 \pm 21.21$ & $866.5 \pm 7.78$ & $863.5 \pm 12.02$ \\
\hline & $\mathrm{R}$ & $854 \pm 28.47$ & $864.4 \pm 4.16$ & $870 \pm 9.82$ & $865.2 \pm 10.94$ & $861.8 \pm 5.59$ \\
\hline & $\mathrm{T}$ & $865.57 \pm 14.91$ & $858.43 \pm 14.64$ & $865.29 \pm 9.3$ & $864.43 \pm 10.71$ & $866.57 \pm 6.58$ \\
\hline \multirow{3}{*}{ LyE } & $\mathrm{p}-\mathrm{DCD}$ & $4.59 \pm 4.3$ & $8.55 \pm 3.5$ & $4.5 \pm .59$ & $5.9 \pm 5.9$ & $14 \pm .75$ \\
\hline & $\mathrm{R}$ & $5.98 \pm 3.3$ & $3.5 \pm 3.28$ & $6.64 \pm 6.67$ & $6.45 \pm 7.03$ & $6.48 \pm 4.77$ \\
\hline & $\mathrm{T}$ & $7.32 \pm 1.94$ & $5.73 \pm 5.11$ & $4.55 \pm 3.94$ & $6.76 \pm 2.8$ & $8.81 \pm 3.81$ \\
\hline \multirow{3}{*}{ Entropy } & $\mathrm{p}-\mathrm{DCD}$ & $4.12 \pm .08$ & $3.31 \pm 1.46$ & $4.5 \pm .6$ & $4.23 \pm .85$ & $3.2 \pm .41$ \\
\hline & $\mathrm{R}$ & $4.48 \pm 0.9$ & $4.51 \pm 0.5$ & $4.68 \pm .3$ & $4.45 \pm .63$ & $4.31 \pm .34$ \\
\hline & $\mathrm{T}$ & $4.4 \pm .6$ & $4.45 \pm .68$ & $4.73 \pm .47$ & $4.37 \pm .36$ & $4.02 \pm .29$ \\
\hline
\end{tabular}




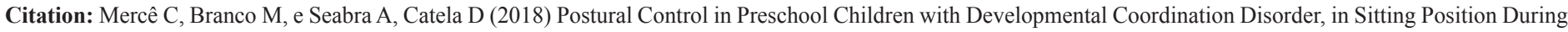
a Functional Task. Yoga Phys Ther Rehabil: YPTR-157. DOI: 10.29011/ ISSN: 2577-0756. 000057

\begin{tabular}{|c|c|c|c|c|c|c|}
\hline \multirow{3}{*}{ Sample Entropy } & $\mathrm{p}-\mathrm{DCD}$ & $.009 \pm .01$ & $.04 \pm .04$ & $.005 \pm .002$ & $.003 \pm .0009$ & $.016 \pm .01$ \\
\cline { 2 - 7 } & $\mathrm{R}$ & $.02 \pm .01$ & $.006 \pm .01$ & $.003 \pm .004$ & $.006 \pm .006$ & $.0033 \pm .003$ \\
\cline { 2 - 7 } & $\mathrm{T}$ & $.03 \pm .03$ & $.03 \pm .03$ & $.012 \pm .02$ & $.006 \pm .005$ & $.01 \pm .009$ \\
\hline
\end{tabular}

Table 9: Descriptive statistical for nonlinear variables for $\mathrm{C} 7$ point.

The present research was, according to our search, the first to use nonlinear methods to analyze the PC in DCD. However, and being the first in this specific area, it was encountered a few problems in the nonlinear data application. We believe that the nonlinear measurements did not reveal more behavior patterns and significant differences between groups due to the data collection, which provide small time series. And not for not being suitable for children with motor disorders, which was already proved in $\mathrm{CP}$ $[11,12,23]$. Reducing the size of all-time series so that they are the same size as the smaller [18] and applying the power spectrum was an essential part if the data treatment [11], and despite collecting the initial data at a high-speed $(240 \mathrm{~Hz})$ data analysis were done with a time series of 900 data points each. This is a larger number than 200, the minimum to calculate SampEn [18], but still far from the 2000 data used by other authors who calculated nonlinear measurements in this kind of study $[11,23]$.

\section{Conclusions}

For all children groups, it was noted postural control changes between conditions, however, no significant differences were found, probably due to the small sample size. The dynamical error of APAS was measured and it revealed excellent values of reliability for the sagittal and frontal plane of motion.

The visual information revealed as an important part of p-DCD postural control, the greater the complexity of the task, the fewer and slower the oscillations were. When removing visual information, $\mathrm{p}$-DCD children reduced their oscillations and velocity for both points, and become also less recurrent, periodic (mean line), stable (maxline) and complex (entropy). Possibly they freeze more degrees of freedom in order to respond to absence of external information.

P-DCD seem to be more dependent on external stimulus to auto organize their own balance, despite of supposedly the task of DEC (doing the plasticine ball with eyes closed) being more difficult than SEC (just be sitting with eyes closed), in SEC p-DCD revealed the smallest and slowest oscillations and simultaneously the most recurrent (higher REC in V and C7) and periodic (higher mean line in V). Probably, because of not having the proprioceptive information on the ball molding they restricted and reduced velocity in their oscillations but become more recurrent and periodic to compensate their balance problems. It seems that the simple fact of performing a functional task can help them to manage their posture. To being true, we should rethink our intervention on these children and focus on functional tasks.
All children groups revealed the same way of action when visual information was removed during a functional task, with less and slower oscillations. In this case, DCD children have the same qualitative postural mechanism as typical. It is likely that the problem p-DCD children have, is not in motor control, but in perception-action cycles' effectiveness [26], where stimulation must be focused.

P-DCD children group revealed, for all children groups (p-DCD, at-risk and typical) and for both points (V and C7), the lowest value for REC, mean line, entropy and SampEn in all conditions (except mean line in SEC and SampEn in SEC and $\mathrm{SD}$ ), and also the lowest maxline in the conditions involving a task for Vertex. Due to having postural control problems p-DCD children become less recurrent (lowest REC), less periodic (lowest mean line) and also simpler and more regular (lowest entropy and highest SampEn).

Looking at LyE, the condition with more stability for p-DCD in both points and at risk for $\mathrm{V}$ was the one with a visual focus, $\mathrm{SD}$, probably due the visual anchor of the researcher molding the ball. The less stable and periodical condition for all groups in both points (except for at risk in C7) was DEC, also in linear data this condition provoked a decrease in amplitude and velocity of the oscillations for all children. So, probably the increased difficulty of the condition forced to all children to oscillate less and slower, having more instability and being less periodic.

In general, the nonlinear analysis demonstrated that at- risk children were the most recurrent (REC) with a tendency to be the most periodic (mean line). Also, linear data revealed these children as the ones with more displacement, amplitude and velocity for all condition except SEO. Maybe for being in a transition zone at risk children oscillate more and faster searching for a motor solution but also in a more recurrent and periodic manner to compensate their difficulties.

Itis possible to use nonlinear methods for analysis of postural control with DCD children. However in the future it is important to find a more suitable strategy to collect data to provide at least of 2000 points of data in time series [11,23], which in turn, nonlinear data can reveal more patterns and highlight other differences between groups.

\section{Conflict of Interest}

The authors declare that do not exist any conflict of interest or any economic interest. 
Citation: Mercê C, Branco M, e Seabra A, Catela D (2018) Postural Control in Preschool Children with Developmental Coordination Disorder, in Sitting Position During a Functional Task. Yoga Phys Ther Rehabil: YPTR-157. DOI: 10.29011/ ISSN: 2577-0756. 000057

\section{References}

1. Association AP (2013) Diagnostic and Statistical Manual of Mental Disorders. (Fifth Edition ed.

2. Vaivre-Douret $L$ (2014) Developmental coordination disorders: state of art. Neurophysiol Clin 44: 13-23.

3. Zwicker JG, Missiuna C, Harris SR, Boyd LA (2012) Developmental coordination disorder: a review and update. Eur J Paediatr Neurol. 16: 573-581.

4. Camden C1, Wilson B, Kirby A, Sugden D, Missiuna C (2015) Best practice principles for management of children with developmental coordination disorder (DCD): results of a scoping review. Child Care Health Dev. 41: 147-159.

5. Vaivre-Douret L1, Lalanne C, Ingster-Moati I, Boddaert N, Cabrol D, et al. (2011) Subtypes of developmental coordination disorder: research on their nature and etiology. Dev Neuropsychol. 36: 614-643.

6. Henderson SE, Sugden DA, Barnett A (2007) Movement Assessment Battery for Children. (Second ed), Psychological Corporation, London (UK).

7. Blank R, Smits-Engelsman B, Polatajko H, Wilson P (2012) European Academy for Childhood Disability (EACD): recommendations on the definition, diagnosis and intervention of developmental coordination disorder (long version). Dev Med Child Neurol 54: 54-93.

8. Macnab JJ, Miller LT, Polatajko HJ (2001) The search for subtypes of DCD: is cluster analysis the answer?. Hum Mov Sci 20: 49-72.

9. Donker SF, Ledebt A, Roerdink M, Savelsbergh GJ, Beek PJ (2008) Children with cerebral palsy exhibit greater and more regular postural sway than typically developing children. Exp Brain Res 184: 363-370.

10. da Costa CS1, Batistão MV, Rocha NA (2013) Quality and structure of variability in children during motor development: a systematic review. Res Dev Disabil 34: $2810-2830$.

11. Deffeyes JE1, Harbourne RT, Kyvelidou A, Stuberg WA, Stergiou N (2009) Nonlinear analysis of sitting postural sway indicates developmental delay in infants. Clin Biomech (Bristol, Avon) 24: 564-570.

12. Deffeyes JE1, Harbourne RT, DeJong SL, Kyvelidou A, Stuberg WA, et al. (2009) Use of information entropy measures of sitting postural sway to quantify developmental delay in infants. Journal of Neuroengineering and Rehabilitation 6.

13. Kyvelidou A, Harbourne RT, Shostrom VK, Stergiou N (2010) Reliability of center of pressure measures for assessing the development of sitting postural control in infants with or at risk of cerebral palsy. Arch Phys Med Rehabil. 91: 1593-1601.
14. Riley MA, Balasubramaniam R, Turvey MT (1999) Recurrence quantification analysis of postural fluctuations. Gait Posture 9: 65-78.

15. Webber C, Zbilut PE (2005) Recurrence Quantification Analysis of Nonlinear Dynamical Systems. In: M.A. Riley and G.C. Van Order, Editors, Tutorials in contemporary nonlinear methods for the behavioral sciences: 26-96

16. Harbourne RT, Stergiou N (2003) Nonlinear analysis of the development of sitting postural control. Dev Psychobiol 42: 368-377.

17. Richman JS, Moorman JR (2000) Physiological time-series analysis using approximate entropy and sample entropy. American Journal of Physiology-Heart and Circulatory Physiology 278: H2O39-H2O49.

18. Yentes JM, Hunt N, Schmid KK, Kaipust JP, McGrath D, et al. (2013) The appropriate use of approximate entropy and sample entropy with short data sets. Ann Biomed Eng 41: 349-365.

19. Adams IL, Ferguson GD, Lust JM, Steenbergen B, Smits-Engelsman BC (2016) Action planning and position sense in children with Developmental Coordination Disorder. Hum Mov Sci 46: 196-208.

20. Smits-Engelsman BC, Blank R, van der Kaay AC, Mosterd-van der Meijs R, Vlugt-van den Brand E, et al. (2013) Efficacy of interventions to improve motor performance in children with developmental coordination disorder: a combined systematic review and meta-analysis. Dev Med Child Neurol 55: 229-237.

21. Mercê C (2016) Recurrence analysis in postural control in children with cerebral palsy. in 3rd IPLeiria's International Health Congress. Leiria.

22. Payton C, Bartlett R (2007) Biomechanical evaluation of movement in sport and exercise: the British Association of Sport and Exercise Sciences guide), Routledge, Abingdon, Oxon; New York, NY.

23. Kyvelidou A, Harbourne RT, Willett SL, Stergiou N (2013) Sitting postural control in infants with typical development, motor delay, or cerebral palsy. Pediatr Phys Ther 25: 46-51.

24. Apthorp D, Nagle F, Palmisano S (2014) Chaos in balance: non-linear measures of postural control predict individual variations in visual illusions of motion. PLoS One 9: e113897.

25. Koo TK, Li MY (2016) A Guideline of Selecting and Reporting Intraclass Correlation Coefficients for Reliability Research. J Chiropr Med 15: 155-163.

26. Chen FC, Tsai CL, Wu SK (2014) Postural sway and perception of affordances in children at risk for developmental coordination disorder. Exp Brain Res 232: 2155-2165.

27. Pincus SM, Goldberger AL (1994) Physiological time-series analysis: what does regularity quantify? Am J Physiol 266: H1643-H1656.

Yoga Phys Ther Rehabil, an open access journal 